\title{
THE IMPACTS OF YOGA AND AEROBIC EXERCISE ON NEURO-COGNITION AND BRAIN STRUCTURE IN EARLY PSYCHOSIS - A PRELIMINARY ANALYSIS OF THE RANDOMIZED CONTROLLED CLINICAL TRIAL
}

Eric Yu Hai Chen, X. Lin, M.M.L. Lam, K.W. Chan, W.C. Chang, G. Joe, G.H.Y. Wong, C.P.Y.

Chiu, P.L. Khong, W.G. Honer, W. Su, C.L.W. Chan, K.F. So, M. Tse

Department of Psychiatry, University of Hong Kong, Hong Kong

Cognitive impairment was detectable in early psychosis together with a decreased hippocampal volume (Wright et al., 2000). These cognitive deficits of attention and memory often result in severe and longstanding functional impairment. Reducing cognitive impairment with pharmacological intervention has not been universally successful (Addington, 2007). The current study aims to explore the effects of aerobic exercise and mind-body exercise (yoga) on cognitive functioning and brain structure for female patients in the early phase of psychosis. We further propose to investigate potential differential effects of these two forms of exercise. 61 female early psychosis patients were recruited from two hospital/clinic sites and randomized into aerobic exercise (walking and cycling), yoga and control groups. Out of the 61 patients, 43 patients completed the 12-week study (16 in yoga group, 12 in exercise group, and 15 in control group). Both interventions were held three times weekly, with each session lasted for an hour. The main outcome measures were structural imaging and neuro-cognitive tests. All participants were assessed both at the baseline and 12 weeks. There was no significant difference in the demographic data at the baseline among three groups. Using ANOVA to compare the changes between the baseline and 12 weeks in three groups, yoga group demonstrated a significant improvement in the verbal encoding $(\mathrm{P}<0.05)$ and semantic clustering $(\mathrm{P}<0.05)$ compared to aerobic exercise and control groups. Both yoga and aerobic exercise groups had a significantly better performance in the DigitSpan test than the control group ( $\mathrm{P}<0.05$ for yoga group, and $\mathrm{P}<0.01$ for aerobic exercise group). Additionally, yoga group had a significant decrease in the omission number of Letter Cancellation Test $(\mathrm{P}<0.05)$ and MADRS total score $(\mathrm{P}<0.05)$, which was not found in the other two groups. Completed MRI data were available in 28 patients. Left hippocampal volume significantly increased $(4 \%, \mathrm{P}<0.01)$ in exercise group after 12 -week intervention, with no change in the control group $(0.3 \%)$. In yoga group, left hippocampal fissure and thalamus proper significantly increased $(\mathrm{P}<0.01)$, and mid-anterior corpus callosum was also found marginally increased $(\mathrm{P}<0.05)$. These preliminary data suggest that the effect of exercise on cognition and hippocampal volume initially found in male chronic schizophrenia patients (Pajonk et al., 2010) can be replicated in female early psychosis patients in Hong Kong. In addition, yoga and aerobic exercise appear to have differential beneficial effects in attention and memory. Yoga improved the semantic encoding and the ability of using learning strategy, while aerobic exercise facilitated more the numeral working memory. Possible increment in the mid-anterior corpus callosum and thalamus proper after yoga intervention may indicate a novel neural mechanism mediating the effect of yoga in psychosis patients.

Reference:

[1] Lin JX, Lam MML, Chan KW, Chang WC, Joe G, Wong GHY, Chiu CPY, Khong PL, Honer $W G$, Su W, Chan CLW, So KF, Tse M, Chen EYH, Addington J (2007). Early Interv Psychiatry, 1(4), 294-307.

[2] Pajonk FG, et al. (2010). Arch Gen Psychiatry, 67(2), 133-143.

[3] Wright IC (2000). Am J Psychiatry, 157(1), 16-25. 